\title{
CLINICAL AND MORPHOLOGICAL INVESTIGATIONS IN A SPONTANEOUS CRYPTOSPORIDIUM ENTERITIS OUTBREAK IN CALVES
}

\author{
I. KALKANOV ${ }^{1}$, I. DINEV ${ }^{1}$, K. DIMITROV $\&$ P. ILIEV ${ }^{2}$ \\ ${ }^{1}$ Department of General and Clinical Pathology, ${ }^{2}$ Department of Veterinary Micro- \\ biology, Infectious and Parasitic Diseases, Faculty of Veterinary Medicine, \\ Trakia University, Stara Zagora, Bulgaria
}

\section{Summary}

Kalkanov, I., I. Dinev, K. Dimitrov \& P. Iliev, 2016. Clinical and morphological investigations in a spontaneous Cryptosporidium enteritis outbreak in calves. Bulg. J. Vet. Med., 19, No 4, 334-339.

\begin{abstract}
The purpose of this report was to present the results of histopathological examination during the course of a natural Cryptosporidium parvum outbreak, as well as the morphology of cells infiltrating affected gastrointestinal tract areas in newborn calves. The study included 18 calves exhibiting a marked diarrhoeic syndrome, between 1 and 8 days of age. Sporulated C. parvum oocysts were demonstrated in faecal smears stained by the method of Henriksen. Coproantigens of $C$. parvum were detected by the rapid Rainbow calf scour 5 BIO K 306 test. Gross lesions were mainly present in the gastrointestinal tract, together with inflammation in regional mesenteric lymph nodes. Microscopic histopathological lesions consisted mainly in desquamative catarrh of intestinal mucosa and intestinal villous atrophy. The affected intestinal epithelium contained multiple Cryptosporidium spp. forms at different stage of the life cycle. The results from histopathological studies of the outbreak allowed confirming some main morphogenetic features of the disease caused by $C$. parvum in newborn and growing calves.
\end{abstract}

Key words: calves, Cryptosporidium parvum, cryptosporidiosis, pathology

Gastrointestinal diseases in newborn and juvenile calves are the commonest problems in this livestock category. The losses are due to high mortality and morbidity rates, reduced weight gain after remission from the disease and disease treatment costs. Neonatal diarrhoea in calves is a multifactorial disease influenced by the breed, feeding regimen and farming conditions. The immunological status of the dams from the herd, farm management and the occurrence of different infectious agents are also important (Bendali et al., 1999; Scott et al., 2004). The risk for occurrence of the disease is the highest during the first month, then decreases as age 
advances (Frank \& Kaneene, 1993; Bendali et al., 1999). At a global scale, neonatal diarrhoea is associated with five primary enteropathogens: Cryptosporidium parvum, rotaviruses, bovine coronavirus (BCV), enterotoxigaenic Escherichia coli (E. coli K99) (Garcia et al., 2000), and Salmonella, all causing diarrhoea. Outbreaks at farms are often observed, especially when the animals originate from different farms with unknown immunological status (Naylor, 2002).

Cryptosporidium spp. (phylum Apicomplexa, family Cryptosporidiidae) are coccidian protozoa affecting the gastrointestinal tract in several mammalian and amphibian species and only the lungs in birds (Helmy et al., 2013). Some of the members of this family possess also well expressed zoo-anthropogenic potential (Castro-Hermida et al., 2002). The possibility for subclinical course and parasite shedding explain the broad geographic spread of cryptosporidiosis. According to Joachim et al. (2003), the prevalence of Cryptosporidium spp. in calves in European countries varies between $20 \%$ and $40 \%$. Together with some bacterial and viral pathogens, Cryptosporidium parvum is considered as one of the most frequent agents of neonatal gastroenterites in calves up to 3 months of age (Chartier et $a l ., 2013)$. The relative death rate is low, but morbidity within the general population in the contact environment is high (Castro-Hermida et al., 2002). The most important clinical sign reported by Joachim et al. (2003) is profuse diarrhoea. Its duration and intensity correspond to the localisation of the infection and the extent of morphological aberrations of the intestinal mucosa. The histological findings of colonised distal small intestine compartments consist in villous atrophy, metaplasia and desquamation of the sur- face epithelium (Fayer \& Xiao, 2007). The authors explained that similar lesions could be also observed in the duodenum, caecum and the colon. In their view, the cell infiltration in lamina propria consisted mainly of neutrophil leukocytes.

The purpose of this report was to establish the histopathological alterations during the course of a natural C. parvum outbreak, as well as the morphology of cells infiltrating affected gastrointestinal tract areas in newborn calves.

The study was conducted on 18 calves with clearly manifested diarrhoeic syndrome aged between 1 and 8 days.

Case history. A sudden increase in mortality rates in calves from the $24^{\text {th }} \mathrm{h}$ of life to 6-8 days of age was reported at a cattle farm housing 200 animals from the Limousin and Hereford breeds, grazing freely on pasture. Clinically, bloody diarrhoea, rapid dehydration and death, without fever, were observed. The palliative general fluid therapy and parenteral treatment with macrolide antibiotics were of no use. At that time, no preventive vaccination in cows have been performed during the dry period.

Coprological samples were obtained manually from ampula recti of each animal and stored at $4{ }^{\circ} \mathrm{C}$ until the analysis. Sporulated oocysts were detected on faecal smears stained by the method of Henriksen (Emmonya Biotech, Chelopech) modified Ziehl-Neelsen staining for acidfast microorganisms. The presence of $C$. parvum coproantigens was confirmed through Rainbow calf scour 5 BIO K 306 (BIOX Diagnostics) rapid test, designed for detection of rotavirus, coronavirus, $E$. coli - F5, Cryptosporidium parvum and Clostridium perfringens type A.

Gross and histological examinations. Eight carcasses were submitted to routine necropsy using the standard protocol. Tis- 
sue samples (size $2.5 \mathrm{~cm}$ ) were collected from the affected gastrointestinal tract areas (abomasum, duodenum, jejunum with mesenteric lymph nodes, ileum, caecum, colon, rectum) for histopathological examination. Samples $(2.5 \times 2.5 \times 1 \mathrm{~cm})$ were also obtained from parenchymal organs - liver, lungs, kidneys, spleen and heart. Specimens for histological examination were fixed in $10 \%$ neutral buffered formalin and embedded in paraffin. From paraffin blocks, $4 \mu \mathrm{m}$ cross sections were cut on a Leica RM 2235 microtome and conventionally stained with haematoxylin-eosin.

The external examination of calf carcasses revealed a strong dehydration and staining of the perianal areas with diarrhoeic faeces. Gross lesions were observed predominantly in the gastrointestinal tract, together with reactive regional mesenteric lymph nodes. They were enlarged 1-2 times, hyperaemic, and haemorrhages were present in some of them. Along the entire intestinal wall, multiple subserous petechial haemorrhages could be perceived and at some areas, single haematomas were seen on the mesentery (Fig. 1). The content of all small intestinal compartments and the caecum was watery, with yellow-greenish colour, mixed with gas bubbles and milk coagula (Fig. 2). The intestinal mucosa was oedematous, hyperaemic, spattered with haemorrhages, and in some loci, especially in the caecum, erosive lesions were present. There were no macroscopic alterations in the other studied visceral organs.

Faecal smears from tested calves stained by Henriksen exhibited sporulated Cryptosporidium spp. oocysts (Fig. 3). The presence of the etiological agent was confirmed by the rapid Rainbow calf scour 5 BIO K 306 test.

Microscopic histopathological lesions consisted mainly in desquamative catarrh of the intestinal mucosa and intestinal villous atrophy. The villi were with rounded edges, and at some places, were fused. An usual finding was the local or diffuse infiltration of the mucosa and submucosa of some areas of small intestine and the caecum with neutrophil leukocytes. The affected intestinal epithelium contained a lot of developmental forms of Cryptosporidium spp. (Fig. 4).

The performed histopathological investigations on Cryptosporidium enteritis in calves demonstrated the presence of

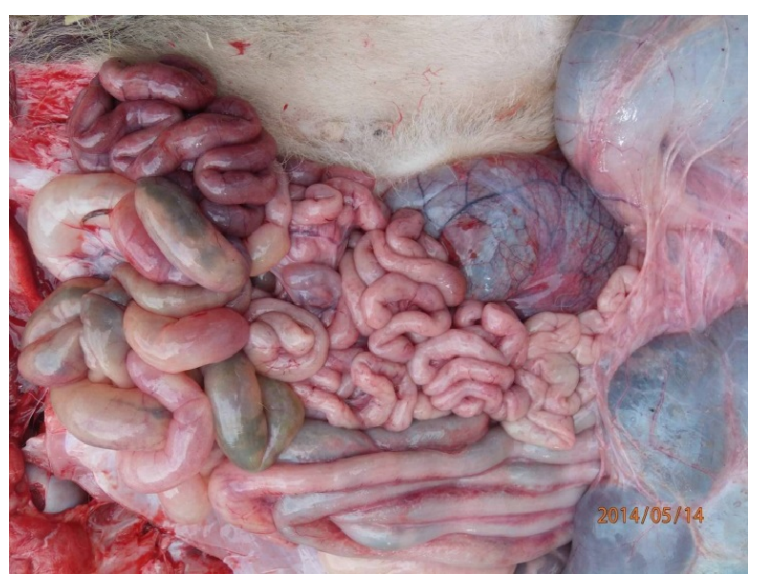

Fig. 1. Catarrhal haemorrhagic enterocolitis - a typical gross finding. 


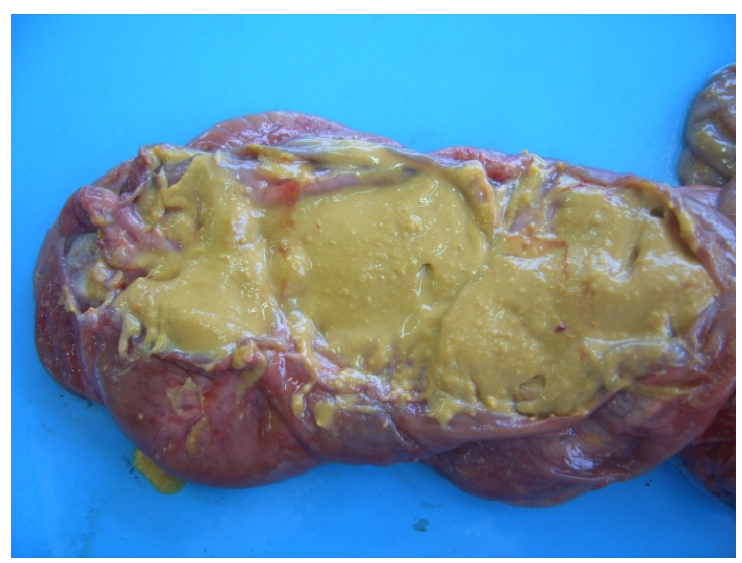

Fig. 2. Specific yellow-greenish content mixed with milk coagula, oedema and hyperaemia of caecal mucosa.

Cryptosporidium spp. in $100 \%$ of animals along the entire intestinal tract. The observed intensive loss of intestinal villi, especially in the distal small intestinal compartment, is associated exactly with established extensive Cryptosporidium in fection (Rosales et al., 1998). At the same time, we believe that the observed signs of regeneration and repair, mainly under the form of crypt hyperplasia in about one quarter of cases ( 5 calves) are an attempt for restoration of mucosal cell barrier, although at an insufficient extent. This fact, as well as the reported high-degree atrophy of intestinal villi predispose to secondary complications, facilitating the colonisation of other pathogens (Argenzio et al., 1990). The hypothesis is confirmed by the results from a number of animal experimentations (Tzipori et al., 1982; Heine et al., 1984; Argenzio et al., 1990; Moore et al., 1995; Gookin et al., 2002).

The intensive loss of enterocytes is attributed to the pathogenetic mechanism of Cryptosporidium infection, namely formation of parasitic vacuole after penetration of the agent into the cytoplasm (de Graaf et al., 1990; O’Handley \& Olson, 2006).
Cryptosporidium infection and neutrophilic infiltration in described case report was established not only in the distal small intestinal compartment, but also in lamina propria of the proximal colon. We agree with the thesis of some researchers that these lesions are at the background of bloody diarrhoea (Jewis et al., 1966) and that in cases when only small intestine is affected and the colon is intact, faeces were free of blood (Jewis et al., 1966; Kovatch \& White, 1972).

The observed outbreak of neonatal calf diarrhoea associated with Cryptosporidium spp. confirms that the pathogen is responsible for a considerable share of gastrointestinal tract diseases in newborn and juvenile calves. We also affirm that the susceptibility to this infection is the highest until the $3^{\text {rd }}$ week of life. Our studies gave us reason to acknowledge, similarly to other research teams, that at this age, the etiology of enterites in calves could be complicated with other agents, mainly of bacterial or viral origin (Chartier et al., 2013). The histological results allowed confirming some main morphogenetic features of Cryptosporidium par- 


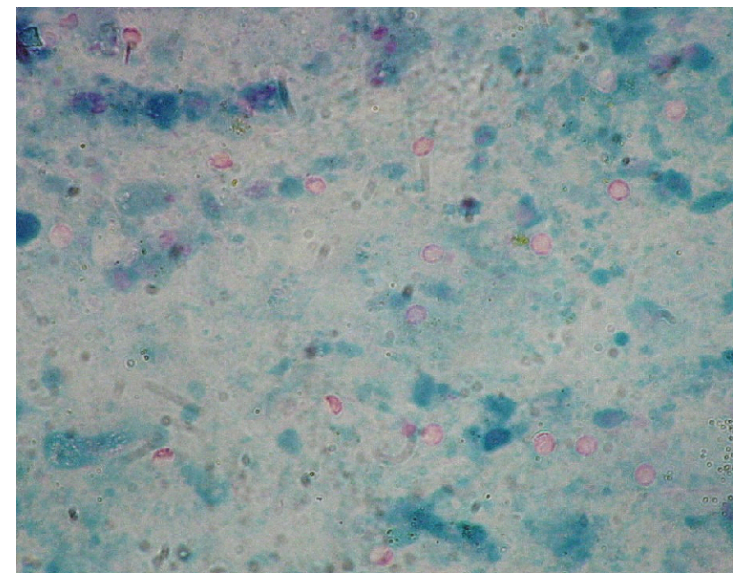

Fig. 3. Cryptosporidium spp. oocysts, faecal smear, Ziehl-Neelsen, $\times 1000$.

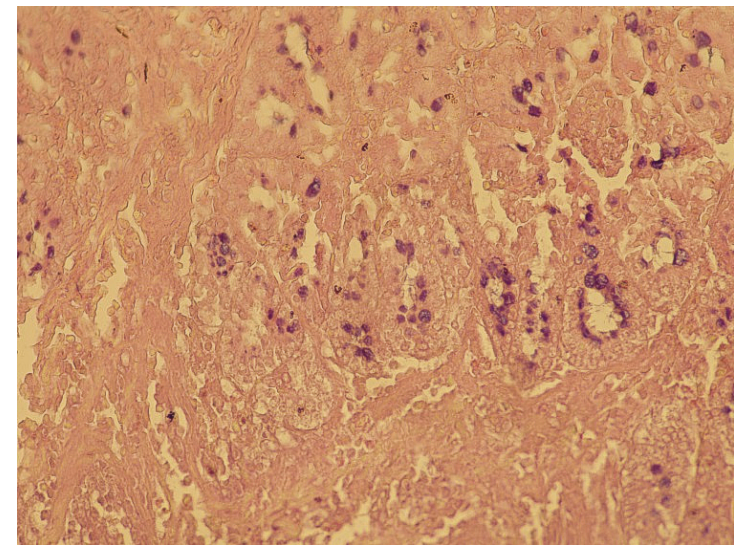

Fig. 4. Multiple developmental forms of Cryptosporidium spp. in mucous ileal crypts, H\&E, $\times 300$.

vum attachment to the surface of enterocytes in the distal small intestine and proximal colon (Gay et al., 2012). We also believe that poor hygienic conditions and production system deficiencies, as well as the insufficient amount of colostrum could be factors for appearance of the disease (Castro-Hermida et al., 2002).

\section{REFERENCES}

Argenzio, A., A. Liacos \& M. Levy, 1990.

Villous atrophy, crypt hyperplasia, cellular infiltration, and impaired glucose-Na ab- sorption in enteric cryptosporidiosis of pigs. Gastroenterology, 98, 1129-1140.

Bendali, F., M. Sanaa, H. Bichet, \& F. Schelcher, 1999. Risk factors associated with diarrhoea in newborn calves. Veterinary Research, 30, 509-522.

Castro-Hermida, A., Y. Gonzales-Losada, M. Mezo-Menendez, E. Aresmazas, 2002. A study of cryptosporidiosis in a cohort of neonatal calves. Veterinary Parasitology, 106, 11-17.

Chartier, C., A. Rieux, A. Delafosse, A. Lehebel \& C. Paraud, 2013. Detection of Cryptosporidium oocysts in fresh calf fae- 
ces: Characteristics of two simple tests and evaluation of a semi-quantitative approach. The Veterinary Journal, 198, 148-152.

de Graaf, A., J.van Dijk \& W. Bovee, 1990. QUALITY: Quantification improvement by converting lineshapes to the Lorentzian type. Magnetic Resonance in Medicine, 13, 343-357.

Fayer, R. \& L. Xiao, 2007. Cryptosporidium and Cryptosporidiosis, $2^{\text {nd }}$ edn, CRC Press, Boca Raton.

Frank, N. \& J. Kaneene, 1993. Management risk factors associated with calf diarrhea in Michigan dairy herds. Journal of Dairy Science, 76, 1313-1323.

Garcia, A., J. Ruiz-Santa-Quiteria, J. Orden, D. Cid, R. Sanz, M. Gomez-Bautista \& R. De La Fuente, 2000. Rotavirus and concurrent infections with other enteropathogens in neonatal diarrheic calves in Spain. Comparative Immunology, Microbiology and Infectious Diseases, 23, 175-183.

Gay, G., T. Courtheoux, C. Reyes, S. Tournier \& Y. Gachet, 2012. A stochastic model of kinetochore-microtubule attachment accurately describes fission yeast chromosome segregation. The Journal of Cell Biology, 196, 757-774.

Gookin, J., S. Nordone \& R. Argenzio, 2002. Host responses to Cryptosporidium infection. Journal of Veterinary Internal Medicine, 16, 12-21.

Heine, J., J. Pohlenz \& H. Moon, 1984. Enteric lesions and diarrhea in gnotobiotic calves monoinfected with Cryptosporidium species. Journal of Infectious Disease, 150, 768-775.

Helmy, M., C. Ballhaus, R. Fonseca, R. Wirth, T. Nagel \& M. Tredoux, 2013. Noble metal nanoclusters and nanoparticles precede mineral formation in magmatic sulfide melts. Nature Communications, 4, 2405.

Joachim, A., T. Krull, J. Schwarzkopf \& A. Daugshies, 2003. Prevalence and control of bovine cryptosporidiosis in German dairy herds. Veterinary Parasitology, 112, 277-288.
Jewis, H., T. Merril \& H. Sprinz, 1966. Coccidiosis in the guinea pig small intestine due to a cryptosporidium. American Journal of Veterinary Research, 27, 408-414.

Kovatch, R. \& J. White, 1972. Cryptosporidiosis in two juvenile rhesus monkeys. Veterinary Pathology, 9, 426-440.

Moore, R., S. Tzipori \& J. Griffiths, 1995. Temporal changes in permeability and structure of piglet ileum after site-specific infection by Cryptosporidium parvum. Gastroenterology, 108, 1030-1039.

Naylor, M., 2002. Neonatal ruminant diarrhea In: Large Animal Internal Medicine, $3^{\text {rd }}$ edn, ed B. P. Smith, Mosby, pp. 352-366.

O'Handley, R. \& M. Olson, 2006. Giardiasis and cryptosporidiosis in ruminants. Veterinary Clinics of North America: Food Animal practice, 22, 623-643.

Rosales, M., T. Arrnedo \& C. Mascaro, 1998. Ultrastructural details of Cryptosporidium parvum development in calf intestine. Memórias do Instituto Oswaldo Cruz, 93, 847-850.

Scott, C., O. Schuldiner \& P. Neufeld, 2004. Role and regulation of starvation-induced autophagy in the Drosophila fat body. Developmental Cell, 7, 167-178.

Tzipori, S., K. Angus \& I. Campbell, 1982. Experimental infection of lambs with Cryptosporidium isolated from a human patient with diarrhoea. Gut, 23, 71-74.

Paper received 22.04.2015; accepted for publication 15.06.2015

\section{Correspondence:}

Ismet Kalkanov, DVM

Department of General and Clinical Pathology Faculty of Veterinary Medicine, 6000 Stara Zagora, Bulgaria, e-mail: ismet_88@abv.bg 